\title{
Camel-Hump T-Wave, Tee-Pee Sign, and Wavy Triple Sign (Yasser's Sign) with Hypocalcemia and Hyperkalemia in Covid-19 Pneumonia with Lacunar Infarction
}

\author{
Yasser Mohammed Hassanain Elsayed \\ Critical Care Unit, Al-Rodah Central Hospital, Damietta Health Affairs, Egyptian Ministry of Health (MOH), Damietta, Egypt.
}

Corresponding Author: Yasser Mohammed Hassanain Elsayed, Critical Care Unit, Al-Rodah Central Hospital, Damietta Health Affairs, Egyptian Ministry of Health (MOH), Damietta, Egypt.

Received date: December 18, 2021; Accepted date: December 27, 2021; Published date: January 07, 2022

Citation: Elsayed Y M H, (2022). Camel-Hump T-Wave, Tee-Pee Sign, and Wavy Triple Sign (Yasser's Sign) with Hypocalcemia and Hyperkalemia in Covid-19 Pneumonia with Lacunar Infarction. J Thoracic Disease and Cardiothoracic Surgery, 3(1); DOI:10.31579/2693-2156/036

Copyright: (c) 2022 Yasser Mohammed Hassanain Elsayed, This is an open access article distributed under the Creative Commons Attribution License, which permits unrestricted use, distribution, and reproduction in any medium, provided the original work is properly cited.

\begin{abstract}
Rationale: A novel COVID-19 is a multi-systemic critical worldwide pandemic infection. Certainly, associated multiple electrolytes imbalance in COVID-19 pneumonia is a remarkable decisive event. Camel-hump T-wave, Tee-Pee sign, and Wavy triple sign (Yasser's sign) are novel highly significant descriptive electrocardiographic signs that are seen in calcium and potassium disturbance. There is an established and strong relationship between and electrocardiographic abnormalities and electrolytes imbalance. COVID-19 pneumonia and cerebrovascular stroke are commonly seen in a patient with Coronavirus infection.

Patient concerns: A 69-year-old married worker Egyptian male patient was presented to the emergency department with COVID-19 pneumonia and cerebrovascular stroke.

Diagnosis: COVID-19 pneumonia with lacunar infarction, hypocalcemia, and hyperkalemia.

Interventions: Chest CT scan, brain CT scan, electrocardiography, oxygenation, and echocardiography.

Outcomes: Initial bad and deterioration outcome but, the dramatic outcome had happened after later management.

Lessons: The understanding of electrocardiographic signs regarding metabolic disorders such as electrolytes imbalance and other associated systemic diseases is very important. Elderly male sex, heavy smoker, COVID-19 pneumonia, cerebrovascular stroke, chronic renal impairment, ischemic heart disease, hypokalemia, hypocalcemia, and hypernatremia represent bad prognostic points and is indicating a high-risk condition.
\end{abstract}

Keywords: COVID-19; camel-hump T-wave; tee-pee sign; wavy triple sign (yasser's sign); hypocalcemia; hyperkalemia; lacunar infarction; cerebrovascular stroke

\section{Introduction}

Generally, in medical diseases, the clinical response is commonly parallel to laboratory and electrocardiographic improvement. A direct relationship between clinical status, ECG, and laboratory workup is essential [1]. Early studies had recorded different electrolyte disturbances at admitted patients with severe COVID-19 infection. As electrolyte imbalance mainly affects the impact of patient care rather than the pathophysiology of COVID-19 [2]. Periodic measuring of electrolytes after the initial presentation during hospitalization is a target to timely establish and appropriate corrective management [2], The relationship between electrolytes disturbance and coronavirus disease 2019 (COVID-19) in patients of the emergency department (ED) is still vague [3]. The role of the ECG in the assessment and management of acutely unwell patients has long since extended beyond the evaluation of chest pain [4]. Johri et al (2009) reported a case of a combination of hyperkalemia and hypocalcemia resulting in pre-cordial QRS-complex with peaked T-wave, prominent $\mathrm{U}$-wave, and prolongation of the descending limb of the T-wave. This resulted in the Twave overlapping the U wave and they called it the "Tee-Pee Sign" because the shape of the QRS complexes resembled the traditional shape of native American Indians dwelling (Figure 1). The combination of prolongation of both the ST segment and descending limb of the Twave resulted in pseudo-prolongation of the QT interval [5]. Camelhump T-wave is not specific for electrolyte imbalance. The presence of multiple severe electrolyte abnormalities is the cause for this very dramatic camel-hump T-wave, formed by fusion of the T-wave and Giant U-wave [4]. Multiple electrolyte imbalances and have observed dramatic ECG changes of camel-hump T waves and the "Tee-Pee sign". A combination of electrolyte imbalance has not previously been 
reported and this is the most dramatic example of camel hump T-wave [5]. Wavy triple an electrocardiographic sign (Yasser's sign) is a new specific diagnostic sign seen in $97.3 \%$ of the cases of hypocalcemia. Wavy triple an electrocardiographic sign can be used as a therapeutic guide in the cases of hypocalcemia [6]. Coronavirus disease 2019
(COVID-19) evolved quickly into a global pandemic with myriad systemic complications, including stroke. COVID-19 is an independent risk factor for stroke in hospitalized patients and mortality, and stroke presentations are frequently atypical [7].

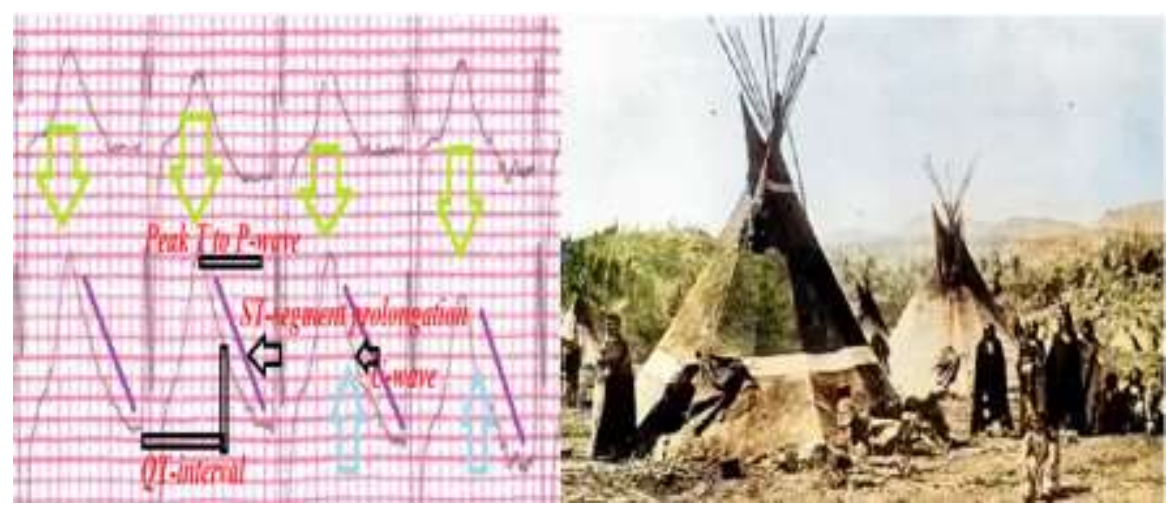

Figure-1 showing traditional shape of native American Indians dwelling.

\section{Case presentation}

A 69-year-old worker, married male, Egyptian, heavy smoker patient was presented to the emergency department (ED) with tachypnea, leftside heaviness, generalized body aches, and palpitations. Fatigue, dry cough, anorexia, and loss of smell were the associated symptoms. There is a recent contact with a confirmed case of COVID-19 pneumonia. He was presented to the ED within 5 days of the above symptoms. He gives a history of chronic renal impairment. He is smoking about 20 cigarettes for 17 years. The patient denied a history of cardiovascular diseases, the same attack, drugs, or any other special habits. Informed consent was taken. Upon general physical examination; generally, the patient was tachypneic, distressed, with a regular pulse rate of VR; $120 \mathrm{bpm}$, blood pressure (BP) of $150 / 90 \mathrm{mmHg}$, respiratory rate of $27 \mathrm{bpm}$, the temperature of $37.8{ }^{\circ} \mathrm{C}$, and pulse oximeter of oxygen $(\mathrm{O} 2)$ saturation of $87 \%$. There is left-side hemiparesis with GCS; 12 . Tests for latent tetany were elicited. Initially, the patient was treated with $\mathrm{O} 2$ inhalation by $\mathrm{O} 2$ cylinder $(100 \%$, by nasal cannula, $5 \mathrm{~L} / \mathrm{min})$. The patient was maintained treated with cefotaxime; (1000 mg IV every 8hours), azithromycin (500 mg PO single daily dose), oseltamivir (75 mg PO twice daily only for 5 days), and paracetamol (500 mg IV every 8 hours as needed). SC enoxaparin $80 \mathrm{mg}$ twice daily), aspirin tablet $(75 \mathrm{mg}$, once daily), clopidogrel tablet (75 mg, once daily), and hydrocortisone sodium succinate (100 mg IV every 12 hours) were added. The patient was daily monitored for temperature, pulse, blood pressure, and O2 saturation. The patient was managed in the ICU with COVID-19 pneumonia, ischemic heart disease, lacunar infarction, hyperkalemia, hypocalcemia, and hypernatremia. The initial ECG was done on the ICU admission showing sinus tachycardia VR; 118, ST-segment depression in both high lateral leads (I and, aVL), Camel-hump T-wave (II, III, aVF, and V3-5), Tee-Pee sign with ST-segment prolongation (V3), and Wavy triple sign Yasser's sign of hypocalcemia (V4-6 leads) (Figure 2). The first brain CT without contrast was done during the day of hospital admission showing lacunar infarction (Figure 3A). The first chest CT without contrast was done during the day of hospital admission showing destructive left ground-glass opacities (Figure 3B). Currently, the patient was admitted to the critical care unit with COVID-19 pneumonia, cerebrovascular stroke, chronic renal impairment, and ischemic heart disease. The initial complete blood count (CBC); $\mathrm{Hb}$ was $10.9 \mathrm{~g} / \mathrm{dl}$, RBCs; $4.1 * 10^{3} / \mathrm{mm}^{3}$, WBCs; $19 * 10^{3} / \mathrm{mm}^{3}$ (Neutrophils; $87.5 \%$, Lymphocytes: $8.3 \%$, Monocytes; 4.2\%, Eosinophils; 0\% and Basophils 0\%), Platelets; $174 * 10^{3} / \mathrm{mm}^{3}$. S. Ferritin was high; $494 \mathrm{ng} / \mathrm{ml}$. D-dimer was high $(0.916 \mathrm{ng} / \mathrm{ml})$. CRP was high $(97 \mathrm{~g} / \mathrm{dl})$. LDH was high (538 U/L). SGPT was normal (41 U/L), SGOT was normal (36 U/L). Serum creatinine was high (4.7 $\mathrm{mg} / \mathrm{dl})$ and blood urea was high $(154 \mathrm{mg} / \mathrm{dl})$. RBS was normal (188 $\mathrm{mg} / \mathrm{dl})$. Plasma sodium showed hypernatremia (152 mmol/L). Serum potassium showed hyperkalemia $(5.8 \mathrm{mmol} / \mathrm{L})$. Ionized calcium was low $0.62 \mathrm{mmol} / \mathrm{L})$. The troponin test was negative $(0.03 \mathrm{U} / \mathrm{L})$. CK-MB was normal (11 U/L). The echocardiography was done on the presentation showing mild grade I, diastolic dysfunction with an EF of $63 \%$. Two calcium gluconate ampoules $(10 \mathrm{ml} 10 \%$ over IV over 20 minutes) were given as an emergency dose. Maintenance therapy with IVI calcium gluconate ampoules $(10 \%$ with the rate; $0.5 \mathrm{mg} / \mathrm{kg} /$ hour over IV over 6 hours) was infused. Actrapid insulin was added in 500 $\mathrm{ml}$ of $10 \%$ dextrose $5 \%$ IV infusion. Periodic inhalation nebulizer sets with salbutamol drops were given. COVID-19 pneumonia, ischemic heart disease, lacunar infarction, hyperkalemia, hypocalcemia, and hypernatremia was the most probable diagnosis. The patient was discharged within 7 days of hospital admission nearly, after clinical, electrocardiogram, and workup improvement. The patient was continued on aspirin tablet (75 mg, OD) for three months, longstanding nitroglycerine oral capsules (2.5 mg BID), and calcium with vitamin D oral preparations (OD) for 2 weeks with follow-up. Further cardiac, renal, neurological, and chest follow-up was advised. 

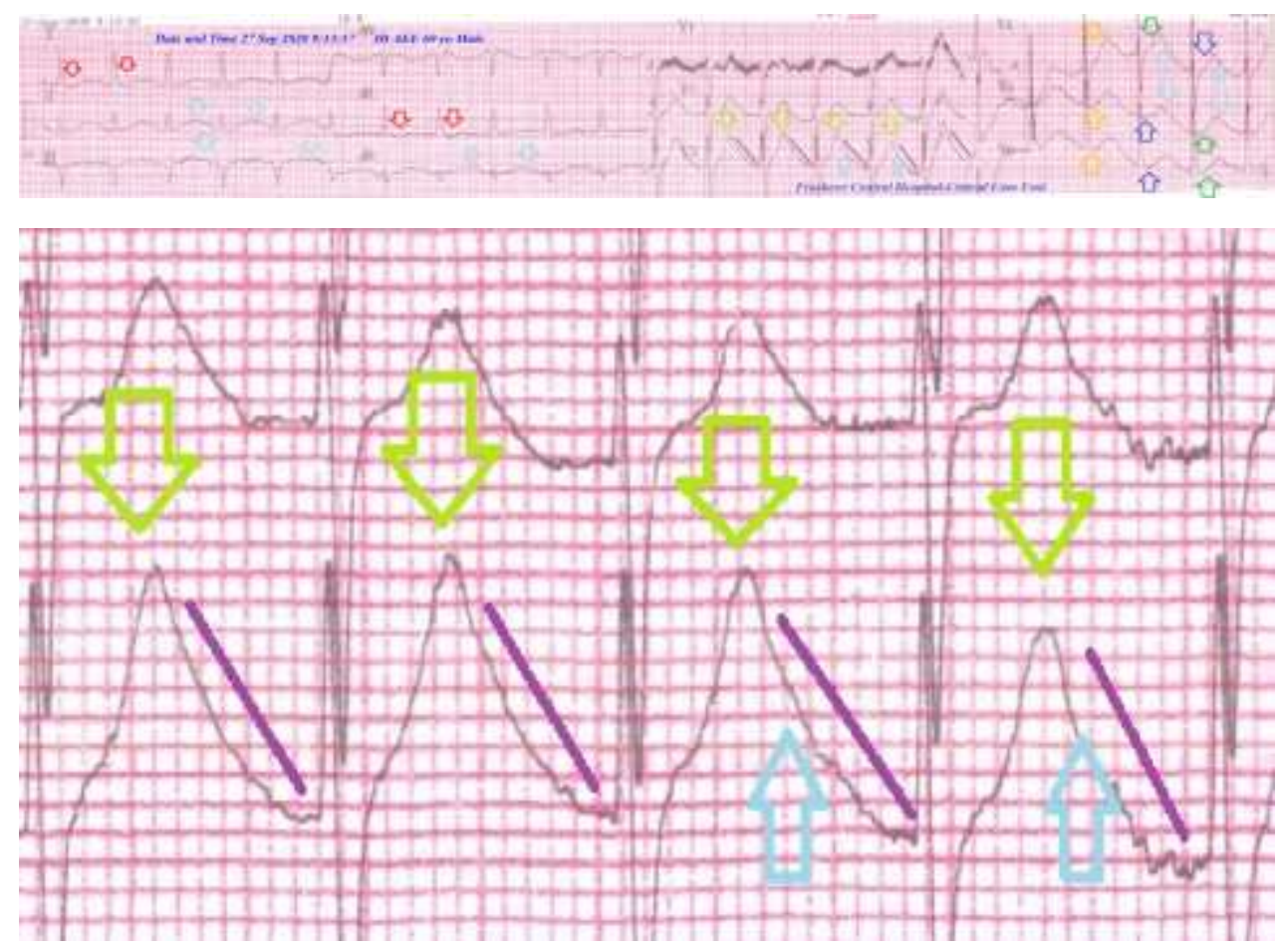

Figure 2: ECG tracing was done on the ICU admission showing sinus tachycardia VR; 118, ST-segment depression in both high lateral leads (I and, aVL; red arrows ), Camel-hump T-wave (II, III, aVF, and V3-5; light blue and lime arrows), Tee-Pee sign with ST-segment prolongation (V3; purple lines), and Wavy triple sign Yasser's sign of hypocalcemia (V4-6 leads; orange, green, and dark blue arrows).

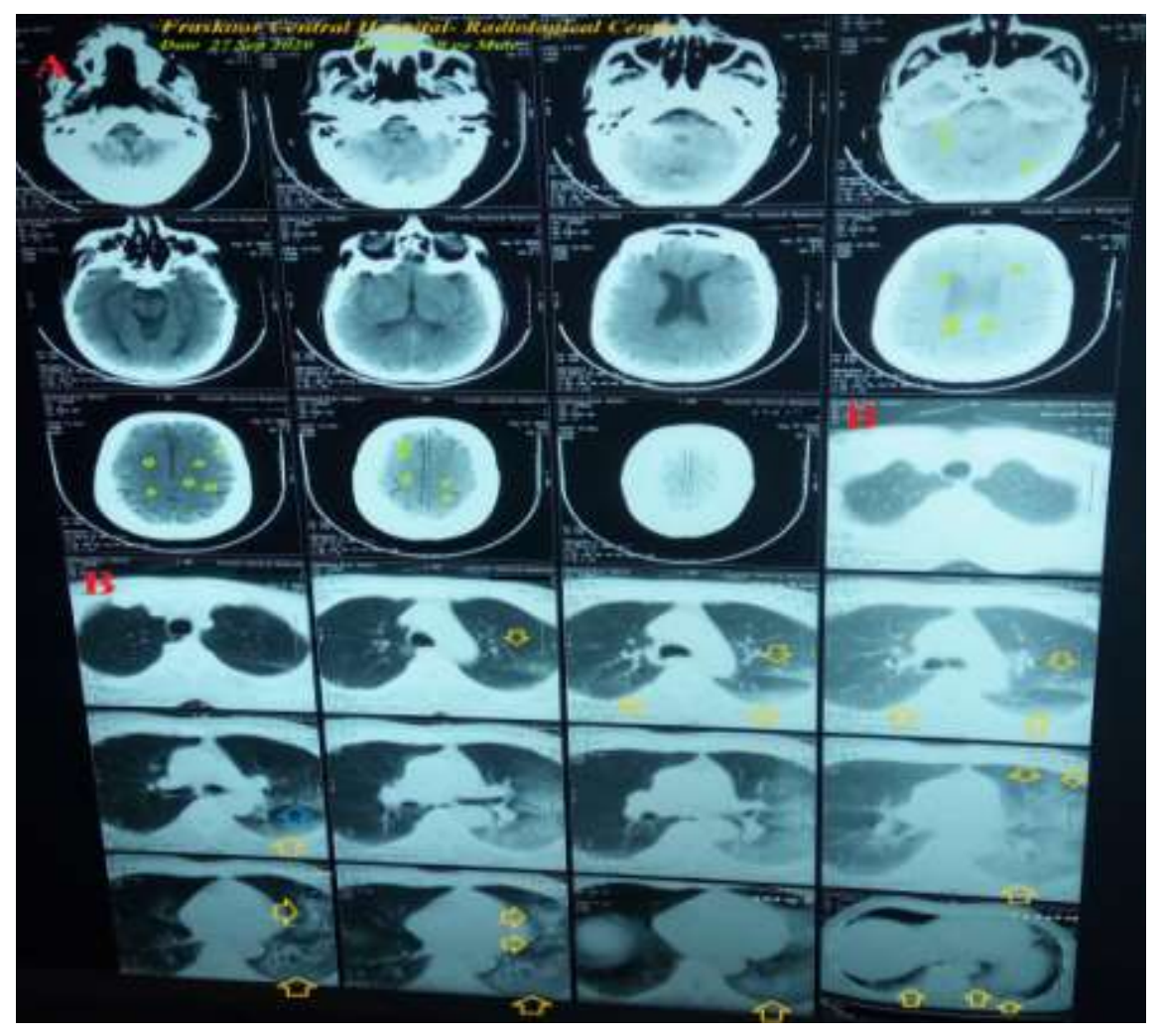

Figure 3(A): Brain CT without contrast was done during the day of hospital admission showing lacunar infarction (lime arrows). (B). Chest CT without contrast was done during the day of hospital admission showing destructive left ground-glass opacities (yellow arrows) with reversed halo sign (blue arrows). 


\section{Discussion}

\section{Overview:}

A 69-year-old married worker Egyptian male patient was presented to the emergency department with COVID-19 pneumonia, ischemic heart disease, lacunar infarction, hyperkalemia, hypocalcemia, and hypernatremia.

The primary objective for my case study was the presence of a patient who presented with COVID-19 pneumonia, ischemic heart disease, lacunar infarction, hyperkalemia, hypocalcemia, and hypernatremia in the ICU.

The secondary objective for my case study was the question of; how did you manage the case at home?

There was a history of contact with a confirmed COVID-19 case. The presence of confirmed COVID-19 case, left-side ground-glass consolidation, and laboratory COVID-19 suspicion on top of clinical COVID-19 presentation will strengthen the COVID-19 diagnosis.

Interestingly, chronic renal impairment with tachypnea due to COVID-19 plays an essential role in the present hyperkalemia and hypocalcemia.

Elderly male sex, heavy smoker, COVID-19 pneumonia, cerebrovascular stroke, chronic renal impairment, ischemic heart disease, hypokalemia, hypocalcemia, and hypernatremia are risk factors.

QTc prolongation was the most probable electrocardiographic differential diagnosis for the current case study. but the QT interval in hypocalcemia rarely exceeds $140 \%$ of the normal ${ }^{5}$.

I can't compare the current case with similar conditions. There are no similar or known cases with the same management for near comparison.

The only limitation of the current study was the unavailability of serial workup.

\section{Conclusion and Recommendations}

- The understanding of electrocardiographic signs regarding metabolic disorders such as electrolytes imbalance and other associated systemic diseases is very important..

- Elderly male sex, heavy smoker, COVID-19 pneumonia, cerebrovascular stroke, chronic renal impairment, ischemic heart disease, hypokalemia, hypocalcemia, and hypernatremia represent bad prognostic points and is indicating a high-risk condition.

\section{Conflicts of interest}

There are no conflicts of interest.

\section{Acknowledgment}

I wish to thank the team nurses of the critical care unit in Fraskour Central Hospital who make extra-ECG copies for helping me. I want to thanks my wife to save time and improving the conditions for supporting me.

\section{Abbreviations}

COVID-19: Coronavirus disease 2019

ECG: Electrocardiogram

ED: Emergency department

ICU: Intensive care unit

$\mathrm{O} 2$ : Oxygen

SGOT: Serum glutamic-oxaloacetic transaminase

SGPT: Serum glutamic-pyruvic transaminase

VR: Ventricular rate

\section{References}

1. Elsayed YMH. (2021) Yasser's COVID-19 Discrepancy Phenomenon: A Novel Phenomenon and Effective Regimen; Retrospective-Observational Study. J Clinical Research and Reports., 8(4); DOI:10.31579/2690-1919/185

2. Lippi G, South AM, Henry BM. (2020) Electrolyte imbalances in patients with severe coronavirus disease 2019 (COVID-19). Ann Clin Biochem. 2020 May;57(3):262-265. DOI: 10.1177/0004563220922255. Epub May 3. PMID: 32266828; PMCID: PMC8173320.

3. De Carvalho H, Richard MC, Chouihed T, Goffinet N, Le Bastard Q, Freund Y, Kratz A, Dubroux M, Masson D, Figueres L, Montassier E. (2021) Electrolyte imbalance in COVID-19 patients admitted to the Emergency Department: a case-control study. Intern Emerg Med. Oct;16(7):1945-1950. DOI: 10.1007/s11739-021-02632-z. Epub 2021 Jan 23. PMID: 33484453; PMCID: PMC7823192.

4. Au Yong JK, Ng JXT, Lynch RM. (2017) Camel Hump T Waves and the "Tee-Pee Sign" - Electrocardiographic Evidence of Severe Electrolyte Abnormalities. Emerg Med Inves. J140.

5. Johri AM, Baranchuk A, Simpson CS, Abdollah H, Redfearn DP. (2009) ECG manifestations of multiple electrolyte imbalance: peaked T wave to $\mathrm{P}$ wave ("tee-pee sign"). Annals of Noninvasive Electrocardiology Apr;14(2):211-214. DOI: 10.1111/j.1542-474x.2009.00283.x. PMID: 19419407; PMCID: PMC6932546.

6. Elsayed YMH. (2019) Wavy Triple an Electrocardiographic Sign (Yasser Sign) in Hypocalcemia. A Novel Diagnostic Sign; Retrospective Observational Study. EC Emergency Medicine and Critical Care (ECEC). 2019;3(2):1-2.

7. Katz JM, Libman RB, Wang JJ, Sanelli P, Filippi CG, Gribko M, Pacia SV, Kuzniecky RI, Najjar S, Azhar S. (2020) Cerebrovascular Complications of COVID-19. Stroke. 2020 Sep;51(9):e227-e231.

DOI: 10.1161/STROKEAHA.120.031265. Epub PMID: 32757751; PMCID: PMC7467046. 


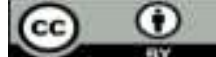

This work is licensed under Creative Commons Attribution 4.0 License

\section{To Submit Your Article Click Here: Submit Manuscript}

DOI: $10.31579 / 2693-2156 / 036$
Ready to submit your research? Choose Auctores and benefit from:

* fast, convenient online submission

* rigorous peer review by experienced research in your field

* rapid publication on acceptance

* authors retain copyrights

* unique DOl for all articles

* immediate, unrestricted online access

At Auctores, research is always in progress.

Learn more www.auctoresonline.org/journals/journal-of-thoracicdisease-and-cardiothoracic-surgery- 